livraisons

d'Histoire

de l'Architecture

\section{Livraisons de l'histoire de l'architecture}

$31 \mid 2016$

La source photographique dans la pratique de I'historien de l'architecture

\title{
La photographie au service de l'enseignement de la construction à l'École des beaux-arts. La démarche de François Vitale
}

The use of photography as an instrument for teaching construction work at the École des beaux-arts. The approach of François Vitale

Die Fotografie als Instrument der Konstruktionslehre an der École des BeauxArts. Das Vorgehen von François Vitale

\section{Amandine Diener}

\section{(2) OpenEdition}

Journals

Édition électronique

URL : http://journals.openedition.org/lha/587

DOI : 10.4000//ha. 587

ISSN : 1960-5994

\section{Éditeur}

Association Livraisons d'histoire de l'architecture - LHA

Édition imprimée

Date de publication : 14 juillet 2016

Pagination : $39-49$

ISSN : 1627-4970

Référence électronique

Amandine Diener, « La photographie au service de l'enseignement de la construction à l'École des beaux-arts. La démarche de François Vitale», Livraisons de l'histoire de l'architecture [En ligne],

31 | 2016, mis en ligne le 14 juillet 2018, consulté le 12 mai 2019. URL : http://

journals.openedition.org//ha/587 ; DOI : 10.4000/lha.587

Ce document a été généré automatiquement le 12 mai 2019

Tous droits réservés à l'Association LHA 


\section{La photographie au service de l'enseignement de la construction à l'École des beaux-arts. La démarche de François Vitale}

The use of photography as an instrument for teaching construction work at the

École des beaux-arts. The approach of François Vitale

Die Fotografie als Instrument der Konstruktionslehre an der École des Beaux-

Arts. Das Vorgehen von François Vitale

\section{Amandine Diener}

1 L'historien et journaliste Georges Toudouze (1877-1972) constate une vulgarisation de la photographie dans l'enseignement au début du $\mathrm{XX}^{\mathrm{e}}$ siècle :

« [Il a quelques années] la projection, sinon inconnue du moins couteuse et réservée aux grands cours supérieurs, n'était point encore admise comme instrument d'enseignement pour les pauvres potaches, [...] mais aujourd'hui il n'en est plus de même, [...] la projection perfectionnée et vulgarisée est devenue accessible à tous et peu coûteuse ${ }^{1}$. »

2 Si les facilités et les bienfaits de l'outil photographique tendent à se démocratiser au cours $\mathrm{du} \mathrm{XX}^{\mathrm{e}}$ siècle, il est intéressant d'interroger comment cela s'est traduit dans l'enseignement de l'architecture à l'École des beaux-arts.

Les grands contours de la scolarité au sein de cet établissement sont connus ${ }^{2}$, ainsi que le système d'apprentissage partagé entre les cours en amphithéâtre et les séances en ateliers $^{3}$. Peu de recherches rendent compte des modalités de cet enseignement. Certains chercheurs se sont intéressés au rôle de l'imprimé dans la formation des élèves architectes ${ }^{4}$, tout en sachant que l'oralité des leçons constitue un obstacle majeur pour qui s'intéresse à l'outil du pédagogue ${ }^{5}$. Mais quel usage fait-on de la photographie ? Estelle un outil didactique? Et à quelles méthodes la collecte des images fait-elle appel ? À quelles fins cette collecte est-elle effectuée? 
4 À partir des archives de l'École des beaux-arts ${ }^{6}$, nous proposons tout d'abord de présenter l'un des objectifs dans la première moitié $\mathrm{du} \mathrm{XX}^{\mathrm{e}}$ siècle: repenser le cours de construction, dont l'actualisation des ressources documentaires, et en particulier la photographie, est l'un des enjeux majeurs. Nous nous intéresserons ensuite à l'enseignement de la construction tel que François Vitale (1898-1962) le dispensait entre 1935 et 1961 ; en effet, ses fonds d'archives contiennent une quantité importante de photographies, de notes de cours et de conférences ${ }^{7}$. Enfin, nous interrogerons la démarche de ce professeur qui, en procédant à une collecte et à un archivage des photographies, aspirait à faire l'histoire de son enseignement.

\section{Les modalités de l'enseignement de la construction en question : l'enjeu d'une actualisation des ressources documentaires}

5 La structuration du cours de construction forme l'un des débats majeurs de l'histoire de l'enseignement de l'architecture au cours du $\mathrm{XX}^{\mathrm{e}}$ siècle ${ }^{8}$. La fin de la Première Guerre mondiale est l'occasion, pour l'École, de s'interroger sur ses propres pratiques. C'est le moment d'améliorer l'enseignement de la construction. Ainsi, en 1917, une commission désignée par le Conseil supérieur de l'enseignement des beaux-arts étudie les mesures nécessaires pour élargir l'enseignement et pour préparer, dans un sens plus pratique, les élèves à l'exercice de la profession.

En 1921, Édouard Arnaud (1854-1943) ${ }^{9}$, alors professeur de construction à l'École des beaux-arts, publie dans L'Architecture une réflexion sur les nouvelles manières d'aborder le cours qui «subit cette année, dans son mode d'enseignement, des modifications profondes ${ }^{10}$.» Consciente de la nécessité de former des architectes après les destructions de la Première Guerre mondiale, l'École est, selon lui, prête à transformer radicalement ses méthodes d'enseignement de la construction. Il suggère: d'abandonner la pratique des cours dictés et accompagnés de dessins que l'élève reproduit sur place, dans l'amphithéâtre, pour laisser place à un mode d'enseignement technique et pratique complet; il préconise d'éliminer tout temps inutilement perdu ; et il suggère de fournir une documentation abondante. Ce procédé, mis en place depuis la prise de poste d'Arnaud en 1920, semble approprié : la projection libère les élèves de la prise de notes et leur permet de porter leur attention sur les diapositives. Des cours imprimés d'environ 2000 pages de textes et 7000 dessins ou vues de chantier sont distribués aux élèves, représentant des schémas de principe, des dessins de procédés de construction ainsi que des vues de chantier et de réalisations en cours d'exécution. Arnaud est toutefois conscient du danger de ces cours imprimés dans le processus d'apprentissage : les élèves ne dessinent plus et perdent une occasion de s'exercer au langage de l'architecture.

7 Parce qu'elle est située au carrefour des sphères scolaire et professionnelle, l'action de François Vitale est intéressante à mesure ${ }^{11}$. Professeur investi dans son métier ${ }^{12}$, il dresse un double constat lorsqu'il est nommé à la succession d'Arnaud en 1935 : l'évolution de l'art de bâtir se traduit par l'arrivée de nouveaux programmes "suivant lesquels doit s'exprimer l'architecture d'aujourd'hui ${ }^{13}$ ", tels que des groupes scolaires, des usines ou des hôpitaux, dont la mise en œuvre requiert de nouveaux matériaux et procédés techniques. Les architectes doivent désormais être formés à ces derniers. Pour permettre de maintenir leur rôle de concepteur-bâtisseur et d'être «à la tête de ce mouvement de 
réforme ", une mise à jour des programmes d'enseignement et des procédés didactiques s'impose. Le cours de construction générale, qui marque la conclusion des études scientifiques à l'École, "contient les éléments dont l'assimilation est essentielle pour exercer la profession d'architecte » et est constitué « d'une documentation indispensable, mais en continuelle évolution ».

\section{François Vitale et les usages de la photographie}

8 En 1932, dans Bâtiri ${ }^{14}$, organe de l'École centrale ${ }^{15}$, Vitale explicite l'importance de la complémentarité du texte et de l'image pour un enseignement efficace de la construction. Conscient des limites de l'attention portée par les élèves durant un cours, il prône un enseignement plus vivant qui illustre concrètement et directement les leçons théoriques promulguées en amphithéâtre. En ces matières, l'enseignement le plus profitable «est celui qui montre.» Il introduit une dimension pratique à l'enseignement de la construction, où les architectes, "intellectuels autant que visuels", saisiront instantanément, grâce à la prise de vue, les théorèmes et principes déclinés précédemment au tableau. La photographie devient ainsi un outil didactique utilisé à des fins pédagogiques. En particulier, la plaque photographique est un outil majeur ${ }^{16}$, comme en témoigne le fonds de plaques de verre dont les positifs en noir et blanc illustrent les cours de constructions civiles confectionnées et héritées d'Arnaud ${ }^{17}$. Les éléments représentés, tels des croquis, des détails techniques, des tableaux d'explications techniques, ou encore des représentations de schémas de composition (ill. 1), illustrent une démarche globale qui assume la gouvernance des principes de la construction par le projet d'architecture. 


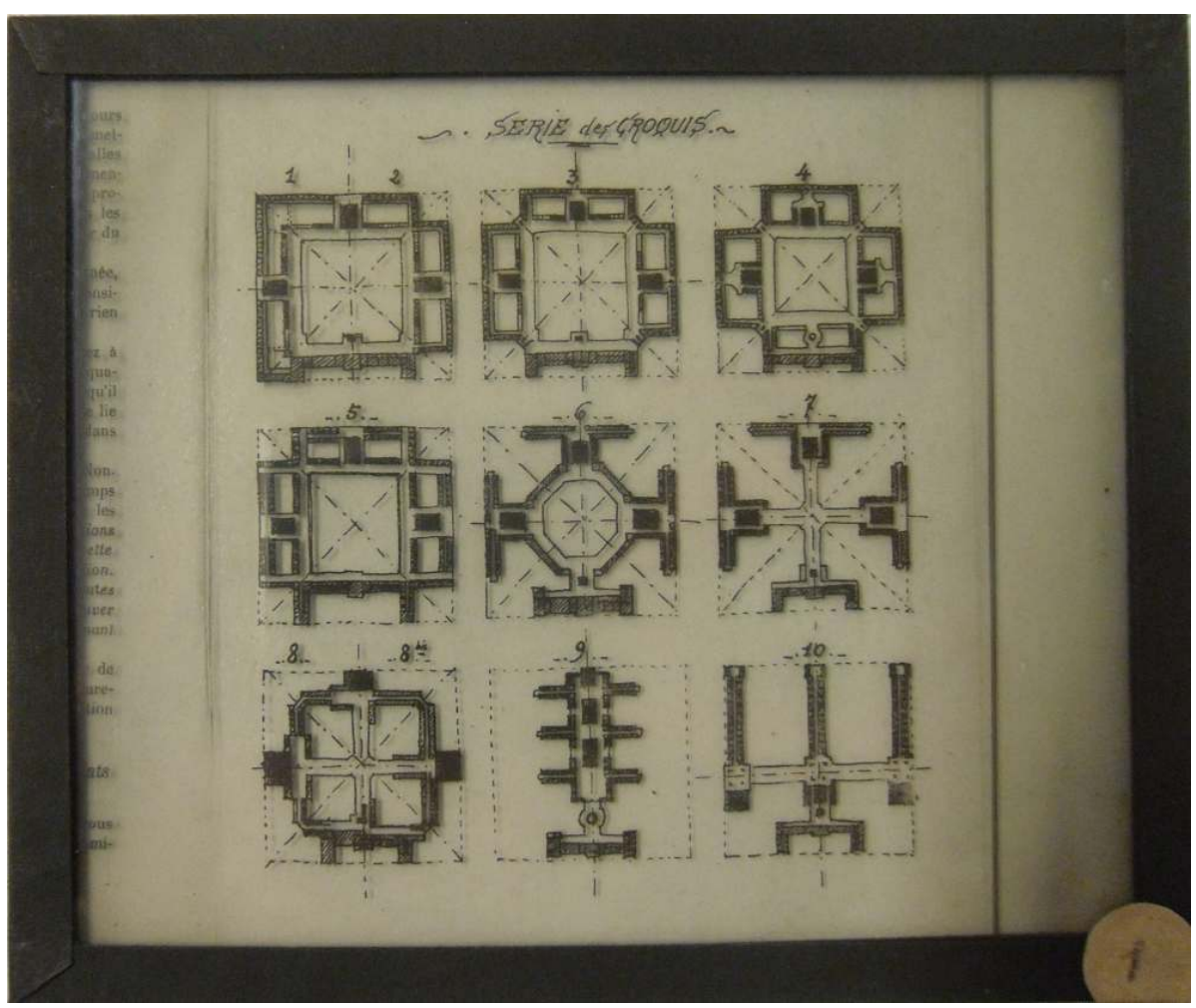

$6 \times 8 \mathrm{~cm}$, dans 186 IFA 406, dossier cours «La Composition » 1 à 16, fonds François Vitale, SIAF/Cité de l'architecture et du patrimoine/Archives d'architecture du XXe siècle.

(c) $\mathrm{Cl}$. Amandine Diener

9 Vitale utilise aussi la photographie comme un vecteur de diffusion des idées lors d'expositions ou de conférences. Ainsi, à l'occasion de l'exposition d'architecture à Caracas pour L'Architecture d'Aujourd'hui en 1950, Jean Fayeton (1908-1968), alors en charge de réunir les documents photographiques sur les récentes réalisations françaises dans le domaine des constructions du travail, demande à Vitale de lui envoyer les photographies d'œuvres qu'il juge dignes de faire figurer dans cette exposition ${ }^{18}$. Il y répond positivement et lui adresse une dizaine de clichés (ill. 2) ${ }^{19}$. Cette même année, Vitale se rend en Belgique pour assurer deux conférences ${ }^{20}$ au Centre belgoluxembourgeois d'information de l'acier. Les correspondances qu'il entretient avec Balbachevsky ${ }^{21}$ témoignent de son assiduité à rassembler « une abondante documentation photographique ${ }^{22}$ ». En amont d'une autre conférence, donnée à la Chambre suisse de la construction métallique en mars 1960, Vitale précise les modalités de son exposé, qui s'appuiera sur " des diapositives 24 x $36 \mathrm{~m} / \mathrm{m}$ encartés dans un carton 50 × $50 \mathrm{~m} / \mathrm{m}^{23}$ » et sur une projection des photographies dont il décline la liste ${ }^{24}$. Enfin, pour un cycle de conférences destiné aux élèves de l'Institut ${ }^{25}$, il prévoit systématiquement des projections d'images. Celles-ci illustrent la variété des exemples choisis pour étayer son propos : la bibliothèque Sainte-Geneviève de Labrouste en 1851 ou la Galerie des machines de Dutert et Sauvestre pour l'Exposition universelle de 1889, la Maison du peuple de Clichy de Lods, Beaudouin, Prouvé et Bodiansky en 1938, ou encore des exemples représentatifs de l'Art déco et de l'architecture moderne comme le Rockefeller Center à New-York. Si ni les textes prononcés ni les négatifs ne sont joints, ces notes illustrent l'éclectisme des exemples choisis par Vitale et la place qu'il accorde à l'image dans ses interventions ${ }^{26}$. 
Dans ses conférences comme dans ses cours, il applique donc le principe énoncé plus haut, qui consiste à accompagner la théorie par l'image pour captiver les auditeurs. Il en est de même dans ses publications, notamment lorsqu'il s'adresse à ses élèves dans la revue Bâtir en privilégiant des croquis effectués au cours de voyage ${ }^{27}$ ou empruntés à Roger Currat $^{28}$, professeur de construction à l'École complémentaire professionnelle de la SICI à Lausanne, qui illustrent des récits de « voyages imaginaires ».

\section{2 : Lettre de François Vitale adressée à Jean Fayeton}

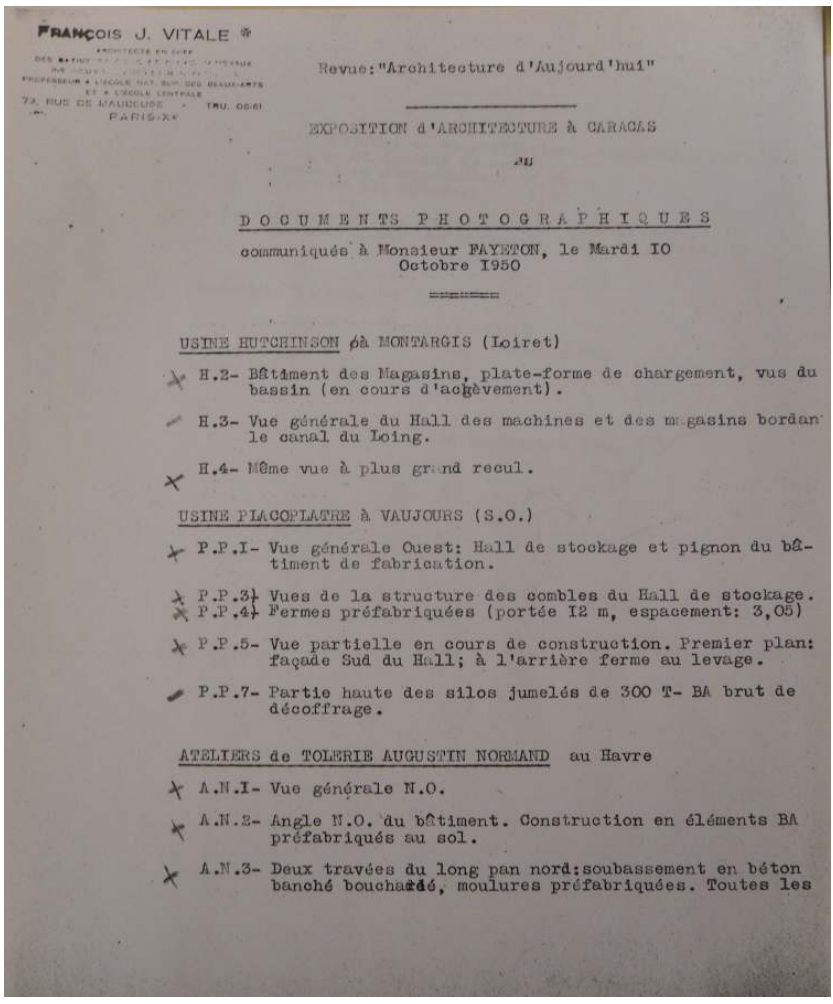

Datée du 10 mai 1950, 1 p., (en réponse à une lettre de Jean Fayeton adressée à François Vitale datée du 30 janvier 1950, 2 p.), dans 186 IFA 22/1, dossier « Notes et correspondances professionnelles, 1929-1961», fonds François Vitale, SIAF/Cité de l'architecture et du patrimoine/Archives d'architecture du XXe siècle.

\section{(c) $\mathrm{Cl}$. Amandine Diener}

À partir de $1944^{29}$, l'École développe des visites de chantier « organisées sous le patronage de M. Untersteller et sous la direction de $\mathrm{M}$. Vitale ${ }^{30}$ ", permettent aux élèves de découvrir de visu des œuvres d'architectes contemporains en cours de construction ${ }^{31}$. Ces moments de prise de contact avec la réalité sont immortalisés par des photographes, comme en témoigne l'importante masse photographique représentant Vitale en compagnie de ses élèves sur des chantiers (ill. 3) ${ }^{32}$. La dimension standard des clichés en noir et blanc $(4,5 \times 6 \mathrm{~cm}$ et $9 \times 6 \mathrm{~cm})$ laisse penser qu'ils ont été pris avec un appareil " petit format ", dont le Leica créé en 1925 est le prototype. Si les photographies utilisées pour illustrer les cours et les conférences sont de natures pédagogiques, celles prises durant les expériences et les visites de chantier n'ont pas la même vocation : l'instantané photographique de ces clichés saisit l'action dans sa vérité, sans mise en scène ni ajustement du décor ${ }^{33}$. 


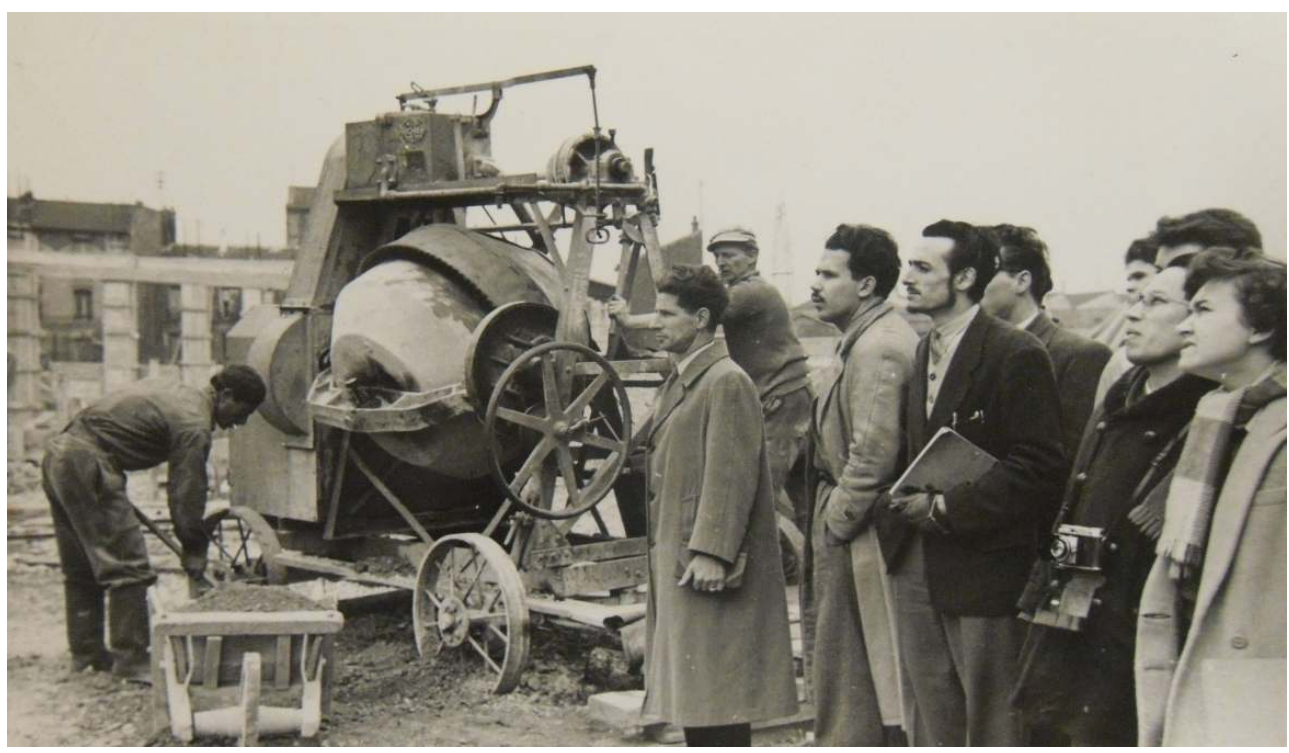

S. d., $8 \times 11 \mathrm{~cm}$, dans 186 IFA 500, dossier « Documents biographiques », pochette 500/3 « Vues de Vitale en compagnie de ses élèves », fonds François Vitale, SIAF/Cité de l'architecture et du patrimoine/Archives d'architecture du XXe siècle.

(c) $\mathrm{Cl}$. Amandine Diener

\section{L'archive photographique ou comment « faire I'histoire » d'un cours}

Le cours de Vitale est constitué en grande partie d'un matériau hérité d'Édouard Arnaud, mais les archives de Vitale dévoilent aussi comment ce dernier a entrepris de constituer un fonds photographique. En outre, il est probable qu'il ait voulu renouveler les outils et les méthodes d'enseignement de la construction à l'École des beaux-arts.

Les documents photographiques récoltés par Vitale sont majoritairement des épreuves envoyées par des élèves lors de voyages en France ou à l'étranger ${ }^{34}$. Certaines correspondances en dévoilent la provenance et l'usage, comme la lettre de Denise Passy stipulant: «Comme vous me l'avez demandé, je vous adresse les négatifs des photos que vous avez remarquées dans mon dossier ${ }^{35}$. » Nous supposons que cette requête a été faite oralement par Vitale à ses élèves durant les cours. Ce projet semble avoir été transmis jusqu'en province, comme en témoigne une lettre de M. Berger, élève de Lyon, qui le " prie d'accepter le don de ces photos et de les considérer comme une contribution aux ouvrages qu['il prépare], trop heureux [lui]-même d'y participer ${ }^{36} »$. Ces épreuves embrassent une grande diversité des sujets photographiés ; l'échantillon consulté met en exergue des clichés de différentes natures : le patrimoine architectural archéologique ${ }^{37}$, la production contemporaine (édifices scolaires ${ }^{38}$, équipements sportifs...), ainsi que les détails de construction ${ }^{39}$ et les procédés de mise en œuvre ${ }^{40}$. (Ill. 4) Certains collègues de Vitale s'associent à cette collecte, tel que Pol Abraham (1891-1966) qui, en mai 1941, lui envoie des photographies de la villa M. J. Rodier à Saint-Cloud ainsi que de l'immeuble d'habitation boulevard Raspail ${ }^{41}$. 


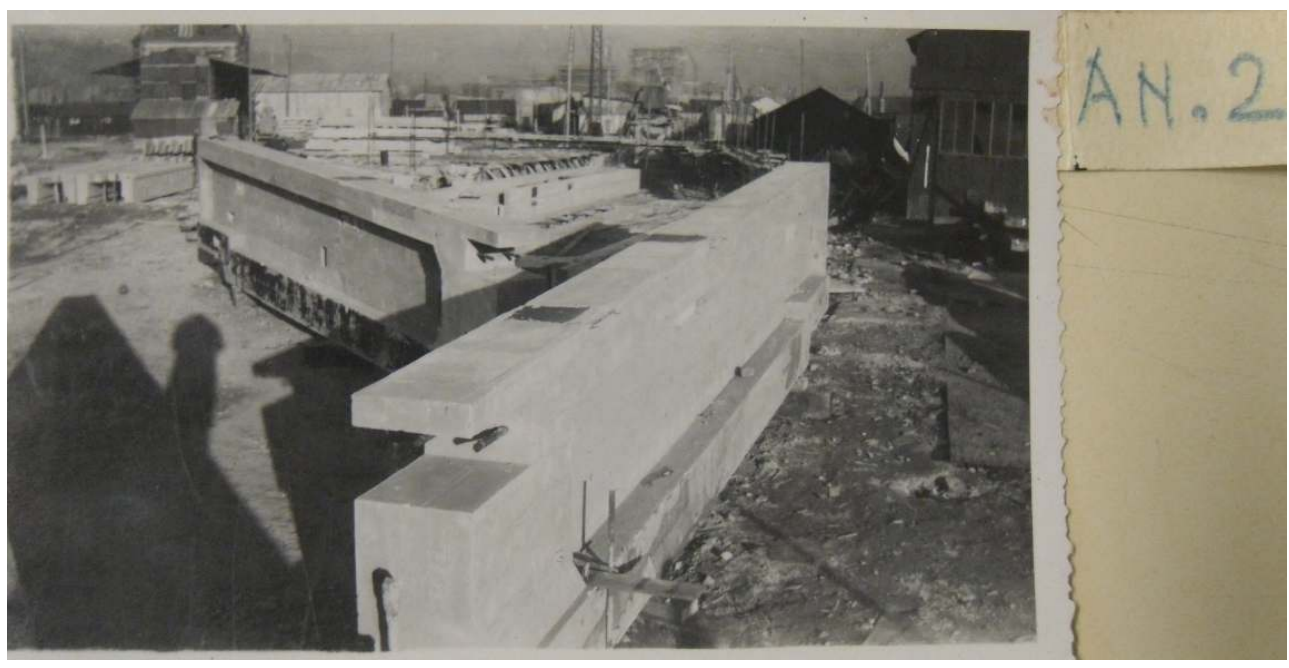

S. d., $9 \times 6$ cm, dans 186 IFA 502, dossier «Ateliers de tôlerie Augustin-Norman, Le Havre, 1945-1955 », pochette 502/1 "Vues de chantiers », fonds François Vitale, SIAF/Cité de l'architecture et du patrimoine/Archives d'architecture du XXe siècle.

(c) $\mathrm{Cl}$. Amandine Diener

D'autres épreuves proviennent de photographes professionnels. Elles ont probablement été rassemblées dans la même intention. Là encore, aucun document ne permet de saisir comment Vitale s'est procuré ces photographies. Hypothèse est faite qu'il aurait demandé aux photographes professionnels l'envoi de clichés montrant des projets dont il avait la charge, ou dévoilant ceux de ses confrères. Les clichés témoignent d'un intérêt essentiellement technique: des détails constructifs et d'exécution ${ }^{42}$, la mise en place d'échafaudages ${ }^{43}$ ou encore des bâtiments conçus à l'aide de nouveaux procédés et techniques de construction ${ }^{44}$. S'il n'y a pas de séries de photographies permettant de restituer l'évolution d'un chantier, ces clichés présentent toutefois l'avantage d'en illustrer certaines étapes clés.

Les cartes postales accumulées par Vitale constituent un autre matériau. Bien que cela puisse, de prime abord, paraître anecdotique, elles révèlent en réalité deux éléments : la lecture en filigrane du réseau socio-professionnel important que Vitale a tissé tout au long de sa carrière, ainsi qu'une illustration de la production architecturale internationale. Certaines cartes sont signées par des collègues, comme l'architecte et ingénieur Édouard Utudjian (1905-1975), en mission d'urbanisme à Bagdad en février 1954, ou encore l'architecte Denys Pradelle (1913-1999), lors d'un congrès au Pays-Bas en juillet 1955. Ce dernier relate avoir fait la connaissance de gens illustres, à l'instar d'André Gutton, et discuté de sujets qualifiés de passionnants, comme un concours d'émulation international. La masse importante des cartes du fonds provient des ÉtatsUnis, d'Angleterre, d'Algérie, d'Italie... et forme un ensemble à la fois homogène et éclectique, puisque les images représentent tant des constructions nouvelles que des fragments d'architecture antique ${ }^{45}$.

15 Le recours à la photographie dans un but didactique à l'École des beaux-arts est perceptible à travers l'enseignement de la construction tel qu'il est renouvelé au $\mathrm{XX}^{\mathrm{e}}$ siècle. Après qu'Édouard Arnaud eut montré l'importance d'une actualisation des ressources documentaires, parmi lesquelles il faut ranger la photographie, François Vitale 
fit de cette dernière un outil indispensable à sa pédagogie. L'étude du rôle de la photographie utilisée dans l'enseignement de Vitale présente un triple intérêt: saisir l'objectif de l'usage du visuel dans le cours de construction, identifier la nature du corpus sélectionné (temporalité, géographie, détails) et comprendre la démarche de ce professeur qui entreprend une collecte et un archivage de documents visuels à vocation pédagogique et documentaire. Le projet de publication d'un nouveau cours imaginé par Vitale, même s'il ne s'est pas concrétisé, aurait été le témoin d'une œuvre collective. Le défi entrepris de renouveler le cours par l'image semble remporté. Il bouscule l'idée reçue selon laquelle l'enseignement de la construction et des techniques serait négligé, voire incomplet, à l'École des beaux-arts. Pierre Saddy, élève de l'École dans les années 1950, se souvient en effet des cours de Vitale pour ses «très belles planches analytiques expliquant les technologies constructives ${ }^{46}$ », et « des efforts [...] pour rendre ses cours attrayants et clairs pour les élèves ».

Pour affiner cette étude, il serait utile de déterminer si d'autres enseignants d'autres disciplines architecturales ont également eu recours à ce même support photographique. Par exemple, l'exposition Marcel Lods, 1891-1978. Photographies d'architecte (1991) a mis en avant la passion de cet architecte-enseignant pour ce médium qui, dans son œuvre, documente tant le chantier que l'aménagement du territoire, notamment grâce à la photographie aérienne ${ }^{47}$. À la faveur de ces exemples, il paraît incontestable que la photographie est déterminante dans l'enseignement de l'urbanisme. Cet enseignement, du reste, a été créé en 1958 sous l'impulsion d'André Gutton (1904-2002), professeur de théorie à l'École des beaux-arts de 1949 à 1957, dont les publications, réunies en plusieurs volumes sous le titre Conversations sur l'architecture ${ }^{48}$, témoignent d'un usage fondamental de la photographie, presque exclusif, où l'image se substitue progressivement au texte. Ainsi, la photographie est riche d'information pour l'historien de l'architecture. Dès lors, son défi en tant que chercheur consiste à aborder conjointement des matériaux et des méthodes issus de deux disciplines à la fois différentes et étroitement liées : l'histoire de l'architecture et celle de la photographie.

\section{NOTES}

1. Georges Toudouze, "L'enseignement scientifique et amusant de l'histoire et de l'histoire de l'art », Le Moniteur de la photographie, $3^{\mathrm{e}}$ série, t. IV, 48 ${ }^{\mathrm{e}}$ année, $\mathrm{n}^{\circ}$ 3, 31 mars 1909, p. 34-35.

2. Citons notamment Arthur Drexler, The Architecture of the École des Beaux-Arts, Museum of Modern Art, New-York, London, Secker \& Werburg, 1977, 544 p.

3. Notamment Guy Lambert, «La pédagogie de l'atelier dans l'enseignement de l'architecture en France aux XIX et $\mathrm{XX}^{\mathrm{e}}$ siècles, une approche culturelle et matérielle », Perspective, $\mathrm{n}^{\circ} 1,2014$, p. 129-136.

4. Notamment Jean-Michel Leniaud, Béatrice Bouvier, Le Livre d'architecture, $X V^{e}-X X^{e}$ siècles: édition, représentations et bibliothèques, Paris, École nationale des chartes, 2002, $335 \mathrm{p}$.

5. Notamment Françoise Waquet, Parler comme un livre: l'oralité et le savoir, $\mathrm{XVI}^{e}-\mathrm{XX}^{e}$ siècles, Paris, Albin Michel, 2003. 
6. Un inventaire des archives de l'École des beaux-arts a été établi dans Brigitte Labat-Poussin, Caroline Obert, Archives de l'École nationale supérieure des beaux-arts, Paris, Centre historique des Archives nationales, 1998, 576 p. Les fonds sont consultables aux Archives nationales sous la série $\mathrm{AJ} / 52$.

7. Les archives de François Vitale sont conservées au Centre d'archives d'architecture du XX siècle, sous la série 186 IFA. Pour cet article, ce sont essentiellement les cartons présentant l'activité d'enseignement de François Vitale qui ont été dépouillés (186 IFA 6, 22, 27, 406, 500, 502, 507,508 à 510).

8. Voir les arrêtés règlementant la section d'architecture de l'École des beaux-arts publiés au J.O. de la République française.

9. Diplômé de l'École centrale de Paris en 1888 puis de l'École des beaux-arts en 1895, Édouard Arnaud mène en parallèle de son activité d'architecte une carrière d'enseignant de construction au sein des deux écoles qui l'ont formé, respectivement entre 1910 et 1934 et entre 1920 et 1934. Ses leçons sont dispensés de 1920 à 1922 à raisons de deux séances par semaine; à compter de 1923, le nombre de leçons passe à trois par semaine. Concernant l'enseignement d'Arnaud, voir notamment Jean Thevenot, Édouard Arnaud et l'enseignement de la construction, thèse de doctorat, Université Paris IV, 1994.

10. Les informations du paragraphe sont issues de Édouard Arnaud, « Le nouvel enseignement de la construction à l'École nationale et supérieure des beaux-arts ", L'Architecture, XXXIV, 1921, n - 11, p. 1.

11. François Vitale est admis à l'École des beaux-arts en 1921 et sort diplômé en 1927. Sa carrière prend un tournant important dans les années 1940, lorsqu'il est nommé chargé de mission au ministère de la Reconstruction et de l'Urbanisme et architecte en chef des bâtiments civils et palais nationaux en 1945, et qu'il rejoint le comité de rédaction de la revue L'Architecture française de 1941 à 1961.

12. Il participe à diverses commissions d'études pour l'évolution de l'enseignement, comme sur «L'enseignement de l'architecture en France (1936-1958)», ou sur «La préfabrication (1941-1954)».

13. Les informations du paragraphe sont issues d'un rapport de 46 pages [s. l.n. d.] traitant de la profession d'architecte et de l'enseignement de l'architecture. Voir 186 IFA 6/2, dossier «Enseignement ENSBA. Rapport sur la création d'un diplôme supérieur d'art plastique. Texte sur la profession d'architecte et l'enseignement de l'architecture ».

14. Tirage de l'article de Vitale, « Visites de chantiers », Bâtir, 186 IFA 28, 7 p., dossier « Articles et conférences de Vitale ».

15. Vitale est maître de conférences de bâtiment et répétiteur à l'École centrale de 1923 à 1961.

16. Notamment Helmut Gernsheim, Alison Gernsheim, The Origins of Photography, New-York, Thames \& Hudson, 1982, $280 \mathrm{p}$.

17. Les plaques de verre utilisées par Vitale sont celles de son prédécesseur et ancien professeur, Édouard Arnaud. La numérotation attribuée par ce dernier a été conservée par Vitale. Voir notamment 186 IFA 406.

18. Lettre de Fayeton à Vitale datée du 30 janvier 1950, 2 p., dans 186 IFA 22, dossier « Notes et correspondances professionnelles ».

19. Trois clichés de l'Usine Hutchinson à Montargis, cinq clichés de l'usine Placoplatre à Vaujours et trois clichés des ateliers de tôlerie Augustin Normand au Havre. Voir la lettre de Vitale à Fayeton datée du 10 mai 1950, 1 p., dans 186 IFA 22, dossier « Notes et correspondances professionnelles ».

20. La première s'intitule «Acier ou Architecture? De l'esthétique des constructions métalliques »; la seconde « Construire en acier. Histoire, ressources, réalisations ».

21. Lettre de Vitale à Balbachevsky du centre belgo-luxembourgeois d'information de l'acier datée du 9 novembre 1950, dans 186 IFA 27 dossier « Articles et conférences de Vitale ». 
22. Lettre de Vitale à Greiner du centre belgo-luxembourgeois d'information de l'acier datée du 28 janvier 1951 de Vitale à Greiner, 1 p., dans 186 IFA 27 dossier «Articles et conférences de Vitale ».

23. Lettre de Vitale à la Chambre suisse de la construction métallique datée du 17 décembre 1959, dans 186 IFA 27/4, dossier « Articles et conférences de Vitale ».

24. Le marché gare de Toulouse, le centre électronique de Bagneux, immeuble d'habitations rue Croulebarbe, le palais de l'Otan, le gymnase d'Ivry à Paris, l'aérogare d'Orly, le bâtiment de la Caisse d'allocations familiales de Paris, la Maison de la radio à Paris et de la centrale thermique de Cachan. Voir la lettre de de Vitale à la Chambre suisse de la construction métallique datée du 4 janvier 1960, 1 p., dans 186 IFA 27/4, dossier « Articles et conférences de Vitale ».

25. «Conférences aux élèves de l'Institut, projections », s. d., 3 p., dans 186 IFA 27, dossier « Articles et conférences de Vitale».

26. La première conférence prévoit quatre projections, la seconde en compte dix-sept, la troisième en compte quatre et la dernière sept.

27. Voir notamment 186 IFA 22/2, dossier «Documentation, notes et correspondances, 1921-1969».

28. 186 IFA 22/3 dossier « Documentation, notes et correspondance professionnelle, 1929-1961 ».

29. "Conférences techniques du cycle des hautes études, 1944 », daté de juillet 1944, signé par François Vitale, dans AJ/52/979, dossier « Conférences particulières techniques ».

30. «Les chantiers ", dans AJ/52/1034, dossier « Grande Masse, participation à la réforme ».

31. Par exemple, en avril 1959 la visite l'aérogare d'Orly avec M. Vicariot (ingénieur en chef des ponts et chaussées), la visite du gratte-ciel $n^{\circ} 1$ avec M. Albert (architecte du projet) et M. Sarf (ingénieur conseil) et la visite du chantier de la Caisse d'allocations familiales, avec $\mathrm{M}$. Longepierre (architectes DESA).

32. 186 IFA 500/3, dossier « Eléments biographiques de Vitale ».

33. André Gunthert, La Conquête de l'instantané. Archéologie de l'imaginaire photographique en France, 1841-1895, thèse de doctorat, EHESS, 1999.

34. Les noms des élèves sont généralement indiqués au dos des clichés.

35. Lettre de Denise Passy à Vitale, s. d., 1 p., dans 186 IFA 507, dossier « Enseignement à l'Ensba, documentation de Vitale ».

36. Lettre de M. Berger à Vitale, s. d., 2 p.

37. Par exemple, trois photographies $(8 \times 11 \mathrm{~cm})$ du Forum de Pompéi, du temple de Poséidon et de la maison d'Herculanum provenant de l'élève nommé Grégoire, dans 186 IFA 510, dossier « Enseignement à l'Ensba, documentation de Vitale ».

38. Par exemple, des photographies $(4 \times 4 \mathrm{~cm})$ de l'École normale supérieure de la rue d'Ulm provenant de l'élève dénommé Degèz, dans 186 IFA 510, dossier «Enseignement à l'Ensba, documentation de Vitale ».

39. Par exemple des photographies $(8 \times 11 \mathrm{~cm})$ du plancher en béton armé et la mise une place d'une ferme d'une usine de bouchon/couronne à Juvisy-sur-Orge provenant de l'élève dénommé Raffi, dans 186 IFA 510, dossier « Enseignement à l'Ensba, documentation de Vitale ».

40. Par exemple, les photographies de la cité universitaire de France outre-mer provenant de l'élève Ragot, dans 186 IFA 510, dossier « Enseignement à l'Ensba, documentation de Vitale ».

41. 186 IFA 508/9, dossier " Abraham Pol : immeuble boulevard Raspail, Paris, VII e arr., 1929 », et 186 IFA 508/10, dossier « Abraham Pol : villa de Jacques Rodier, Saint-Cloud ».

42. Par exemple, le studio $\mathrm{F}$. Harand pour les clichés d'une usine à gaz à Epernay ou encore le studio Henrot pour les clichés du viaduc de Nogent-sur-Marne, dans 186 IFA 507/6, dossier « Enseignement à l'Ensba, documentation de Vitale ».

43. Par exemple, le studio Jean Collas pour la société d'échafaudages Entrepose. Voir 186 IFA 507/6, dossier « Enseignement à l'Ensba, documentation de Vitale ». 
44. Par exemple, la société Baffrey-Hennebique pour les clichés du monument de la Pointe de la Grave, dans 186 IFA 507/6, dossier « Enseignement à l'Ensba, documentation de Vitale ».

45. Par exemple, la carte postale du pont-rail de Nogent-sur-Marne achevé, en écho à la photographie qui le représentait en cours d'exécution, dans 186 IFA 509/5, dossier « Enseignement à l'ensba, documentation de Vitale ».

46. Jacques Gubler, Jean Tschumi, Architecture, échelle, grandeur. Les archives de la construction moderne, Lausanne, Presses polytechniques universitaires romandes, 2008, p. 106.

47. Marcel Lods, Marcel Lods, 1891-1978: photographies d'architecte, catalogue d'exposition, Paris, Centre Georges Pompidou, Académie d'architecture, 1991, n. p. L'exposition est notamment oragnisée par Peter Uyttenhove, suite à sa thèse de doctorat sous la direction d'Hubert Damisch à l'EHESS : Marcel Lods, 1891-1978. Une architecture de l'action.

48. André Gutton, Paris, Éd. Vincent, Fréal \& $\mathrm{C}^{\mathrm{ie}}$ : Conversations sur l'architecture : l'édifice dans la cité , t. 1, 1952, 277 p.; Conversations sur l'architecture: la maison de l'homme, t. 2, 1954, 234 p.; Conversations sur l'architecture: les édifices religieux et culturels, t. 3a, 1956, 415 p. ; Conversations sur l'architecture : l'urbanisme au service de l'homme, t. 6, 1962, 655 p.

\section{RÉSUMÉS}

La photographie se démocratise dans la première moitié du $\mathrm{XX}^{\mathrm{e}}$ siècle. Cet article questionne le rôle joué par ce nouveau ce médium dans les méthodes d'enseignement de la construction à l'École des beaux-arts à cette période. Pour étayer son propos, l'auteure s'appuie, d'une part, sur un corpus d'archives produites par l'École et, d'autre part, sur le fonds important d'images conservées dans les archives François Vitale, professeur de construction actif à l'École entre 1935 et 1961. La première partie de cette étude montre comment l'enseignement de la construction a été repensé dans la première moitié $\mathrm{du} \mathrm{XX}^{\mathrm{e}}$ siècle au prisme de nouvelles ressources pédagogiques, en particulier celui de la photographie. La seconde partie relate plus concrètement comment François Vitale se servait de ce médium durant ses cours. Enfin, la troisième partie s'attache à analyser sa méthode d'enseignement, sensible à travers sa collection de prises de vue réalisées dans le cadre d'un projet de publication de ses cours.

During the $20^{\text {th }}$ century, a process of democratization of photography takes place. This paper asks about the photography was used as an instrument for teaching construction work at the École des beaux-arts. In order to do that, on the one hand we have analysed the archives of the Beaux-arts, on the other hand the large amount of pictures contained in the archives of François Vitale, professor between 1935 and 1961 of Construction. The first part of this paper shows that in the first half of the $20^{\text {th }}$ century the aim of the École des beaux-arts was to update teaching methods of construction work by using new resources, especially the photography. The second part shows how Vitale used this medium for his teaching activity. The last part analysed the approach of Vitale through his collection of pictures, made with the purpose of publishing a new course.

In der ersten Hälfte des 20. Jahrhunderts in eine zunehmende Demokratisierung der Fotografie zu beobachten. Dieser Beitrag hinterfragt die Rolle, die das neue Medium für die Lehrmethoden in der Konstruktionslehre an der École des Beaux-Arts in dieser Zeit spielt. Dabei stützt sich die Autorin einerseits auf Quellenbestände der École selbst, andererseits auf das umfangreiche 
Privatarchiv des ehemaligen Professors für Konstruktionslehre François Vitale, der zwischen 1935 und 1961 an der Hochschule lehrte. Der erste Teil des Artikels zeigt auf, wie sich die Konstruktionslehre in der ersten Hälfte des 20. Jahrhunderts unter Einbeziehung neuer pädagogischer Hilfsmittel wandelte und wie nunmehr insbesondere die Fotografie mit einbezogen wurde. In einem zweiten Teil wird eingehender betrachtet, wie François Vitale selbst sich dieses Mediums in seinem Unterricht bediente. Schließlich beschäftigt sich ein dritter Teil mit einer Analyse seiner Unterrichtsmethode anhand einer Sammlung von Aufnahmen im Rahmen eines Publikationsvorhabens seiner Kurse.

\section{AUTEUR}

\section{AMANDINE DIENER}

Amandine Diener est architecte diplômée d'État. En 2011, elle a soutenu un master à l'École nationale supérieure d'architecture de Strasbourg sur « La société des Amis du Vieux Strasbourg (1957-2010). Histoire, rôles et actions ». Doctorante à l'université de Strasbourg, son sujet de thèse porte sur « L'École des beaux-arts et l'enseignement de la théorie de l'architecture au XX siècle. Évolution d'une discipline et d'une pédagogie : de Julien Guadet, Georges Gromort à André Gutton (1883-1962)» (dir. Anne-Marie Châtelet). Son champ de recherche comprend l'enseignement de l'architecture et l'histoire et les théories architecturales. Elle a récemment collaboré à l'édition d'Anne-Marie Châtelet et Franck Storne, Des Beaux-Arts à l'université. Enseigner l'architecture à Strasbourg (Strasbourg, 2013) et publié un article sur la « Production théorique et diffusion des modèles. De l'École polytechnique à l'École des beaux-arts (1802-1967) » dans la Revista de Arquitectura (vol.17, 2015). Adresse électronique : amandinediener@wanadoo.fr 